\title{
Regulatory Signals Influencing Expression of the PDA1 Gene of Nectria haematococca MPVI in Culture and During Pathogenesis of Pea
}

\author{
Rana Khan ${ }^{1}$ and David C. Straney ${ }^{1,2}$ \\ ${ }^{1}$ Molecular and Cell Biology Program and ${ }^{2}$ Department of Cell Biology and Molecular Genetics, University of \\ Maryland, College Park 20742, U.S.A. \\ Accepted 9 April 1999.
}

\begin{abstract}
The PDA1 gene of the filamentous fungus Nectria haematococca MPVI (anamorph: Fusarium solani) encodes a cytochrome P450 monooxygenase that detoxifies pisatin, the isoflavonoid phytoalexin produced by its host, garden pea (Pisum sativum). PDA1 is regulated by several signals in culture that may control its expression during pathogenesis of pea. It is induced by pisatin and repressed by glucose and amino acids. Deletion analysis was performed on the $P D A 1$ promoter to define regulatory regions, using a $\beta$ glucuronidase (GUS) reporter gene fusion. The results identified a region between -287 and -429 , relative to the start of transcription, that mediated repression by either glucose or amino acids in culture, independent from pisatin induction. Transformants bearing $P D A 1$ promoter constructs displaying altered regulation in response to the different signals were used to infect pea epicotyls in order to correlate regulation in culture with that observed during pathogenesis of the host. Removal of the nutritional response region did not have a major effect on the induction of the promoter observed during growth in pea. However, induced expression in planta was lacking in a $P D A 1:$ :GUS construct that lacked pisatin response in culture. These results suggest that the host-specific stimulus, pisatin, is a primary stimulatory signal for $P D A 1$ regulation during pea pathogenesis.
\end{abstract}

Many fungal and bacterial genes that play a role in plant pathogenesis are not constitutively expressed, but are instead regulated in some manner. The importance of gene regulation in pathogenesis is underscored by the loss of pathogenicity in disruptions of certain common signal transduction components, such as G proteins, cyclic AMP (cAMP)-dependent kinase, and MAP kinase (Kasahara and Nuss 1997; Mitchell and Dean 1995; Xu and Hamer 1996). Identifying the cues and signal pathways that regulate pathogenicity-associated genes should lead to an understanding of how pathogens coordinate the process of pathogenesis.

In several plant-fungal interactions, cues that regulate different pathogenesis-associated genes have been identified.

Corresponding author: D. Straney, Department of Cell Biology and Molecular Genetics, H. J. Patterson Hall, University of Maryland, College Park 20742-5815, U.S.A.; Telephone: 1-301-405-1622; Fax: 1-301314-9082; E-mail: straney@umail.umd.edu
These cues include changes in nutrient levels presumably encountered in the plant (Talbot et al. 1993; Van den Ackerveken et al. 1994), and plant-specific compounds such as pectin or cutin fragments (Gonzalez-Candelas and Kolattukudy 1992; Lin and Kolattukudy 1978). More host-specific cues may regulate many other fungal genes. One pectate lyase gene in Nectria haematococca MPVI displays induced expression during host pathogenesis that cannot be reproduced in culture with cell wall preparations or nutrient starvation (Guo et al. 1996). A number of other plant-induced genes have been found to be induced in planta but not by any defined cultural conditions (Benito et al. 1996; Ehrenshaft and Upchurch 1993; Van den Ackerveken et al. 1992). Presumably, these are induced by as yet unidentified cues in the host.

The pisatin demethylase (PDA1) gene of pea-pathogenic strains of $N$. haematococca MPVI (anamorph: Fusarium solani) offers a good system for the study of plant-induced genes because it is regulated by nutrients levels as well as by an identified host-specific compound encountered in its host, garden pea (Pisum sativum). Pisatin demethylase is a cytochrome P450 monooxygenase that detoxifies pisatin, a fungistatic isoflavonoid phytoalexin produced by garden pea. $P D A 1$ has been shown to have a significant but partial role in pathogenicity on pea in studies of gene disruption (Wasmann and VanEtten 1996) and gene addition (Ciuffetti and VanEtten 1996; Oeser and Yoder 1994; Schäfer et al. 1989). The levels of Pda enzyme and PDA1 mRNA are specifically induced in culture by addition of pisatin (VanEtten and Barz 1981). Since the active isomer of pisatin is produced mainly in garden pea, pisatin would constitute a host-specific signal. Expression of $P D A 1$ is also suppressed in culture by the addition of high levels of glucose or amino acids (Straney and VanEtten 1994). Nutritional regulation of $P D A 1$ could provide a major stimulatory signal during pathogenesis by analogy to the plantinduced genes $A V R 9$ and $M P G 1$, which are induced in culture by nutritional de-repression (Talbot et al. 1993; Van den Ackerveken et al. 1994). Although polymerase chain reaction (PCR) detection of PDA1 expression shows it to be induced during pathogenesis of pea (Hirschi and VanEtten 1996), the relative contributions of host-specific signals (pisatin) or nonspecific signals (nutrients) have not been determined. In addition to the uncertainty of the relative biological roles of pisatin and nutritional regulation, the interdependence of their regulatory pathways is unclear. In previous studies measuring 
regulation of $P D A$ enzyme levels in fungal culture (Straney and VanEtten 1994), it could not be discerned whether nutritional repression acted only to suppress pisatin induction or if it acted independently to reduce both basal and induced expression.

The objectives of the present study were to characterize the nature of nutritional regulation of PDA1 and to determine the relative contributions of nutritional de-repression and pisatin induction toward $P D A 1$ expression during growth within the host plant. We have constructed a $P D A 1$ promoter fusion to the GUS ( $\beta$-glucuronidase) reporter gene in order to measure promoter activity in culture and during growth in the pea plant. This reporter gene system provides more sensitive quantitation of promoter activity in culture than previous studies with pisatin demethylase enzyme activity. Further, the reporter allows quantitation of promoter activity during pathogenesis, expanding upon its previous use for quantitation of fungal growth within the plant with a constitutive promoter (Stahl 1994). Alterations in the PDA1 promoter that remove responses to specific stimuli in culture can then be correlated to changes in expression during pathogenesis on plants. Our results indicate that while the $P D A 1$ promoter is sensitive to

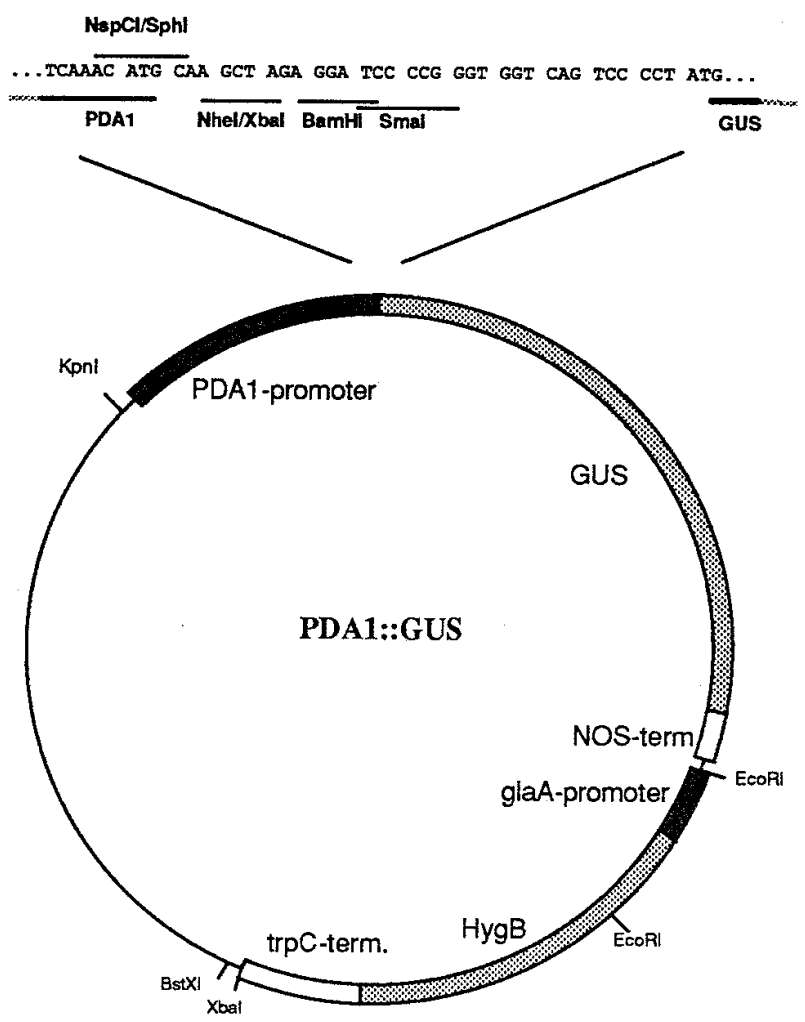

Fig. 1. Reporter gene construct for analysis of $P D A 1$ expression. A 1.1kb promoter fragment of $P D A 1$, including the translated ATG, was fused in frame with the $\beta$-glucuronidase (GUS) reporter gene/NOS terminator from pBI101 (Clonetech, Palo Alto, CA), as described in Materials and Methods. The DNA sequence of the PDA1::GUS junction is shown. The $K p n \mathrm{I} / E c o$ RI fragment was introduced into a pBluescript vector (thin line) carrying the selectable marker, the hygromycin phosphotransferase gene (HygB). HygB is expressed by fusion to the Aspergillus niger glucoamylase $(g l a \mathrm{~A})$ promoter and Aspergillus nidulans trp $\mathrm{C}$ terminator. The insertions replace the $K p n I$ to $X b a \mathrm{I}$ region of the pBluescript polylinker. physiologically relevant levels of glucose or amino acids as repressive signals in culture, the main stimulatory signal for PDA1 during pathogenesis of pea appears to be pisatin induction rather than nutrient de-repression.

\section{RESULTS}

\section{Regulated expression of the $P D A 1$ promoter::GUS reporter gene construct.}

In order to measure $P D A 1$ promoter expression in vivo, a transcriptional fusion was constructed between a 1.1-kb fragment of the PDA1 promoter and the GUS reporter gene. The $P D A 1:: G U S$ fusion was inserted into a vector containing a selectable marker for hygromycin resistance (Fig. 1) and was transformed into $N$. haematococca strains through ectopic integration. Transformation utilized two $N$. haematococca strains: a $\mathrm{Pda}^{+}$strain (77-2-3) carrying the native PDA1 gene and a $\mathrm{Pda}^{-}$strain (94-6-1) lacking the entire chromosome that carries the $P D A 1$ gene.

To validate the use of the fusion construct in promoter analysis, it was first determined whether the GUS reporter gene under control of the $P D A 1$ promoter responded to regulatory signals in the same way as the promoter of the native $P D A 1$ gene. When assayed in culture, a $P D A 1::$ GUS transformant of 77-2-3 displayed similar patterns of expression of both GUS and Pda enzyme activities; mycelium in phosphate buffer alone produced a low level of activities while addition of pisatin induced these activities 10- to 20fold within 10 to $12 \mathrm{~h}$ of its addition (Fig. 2). Supplementation of $270 \mathrm{mM}$ glucose or $166 \mathrm{mM}$ casamino acids to the mycelia in phosphate buffer, with or without pisatin treatment, produced the background level of Pda activity seen in phosphate buffer alone in the absence of pisatin, as characterized previously for this strain (Straney and VanEtten 1994). Glucose or casamino acid treatment also reduced GUS expression in either the presence or absence of pisatin.

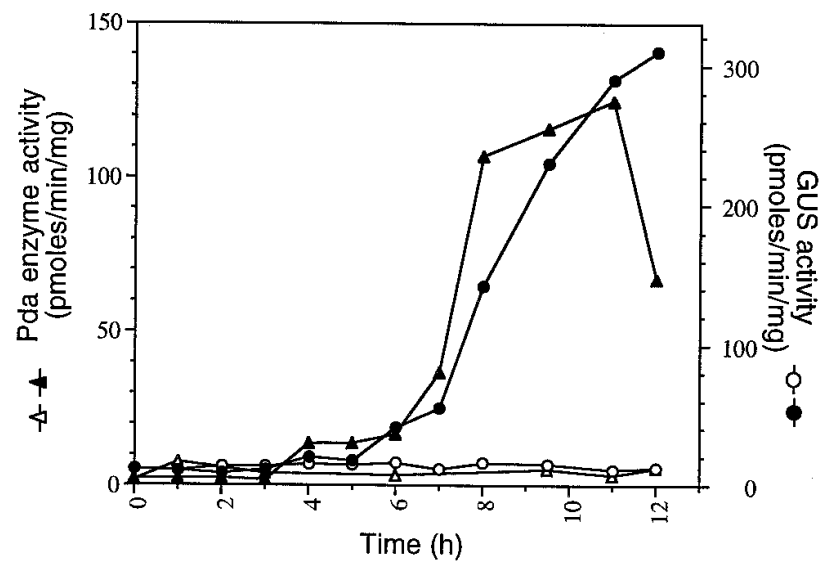

Fig. 2. Induction of the $P D A 1$ enzyme and $\beta$-glucuronidase (GUS) activity in transformants. A full-length PDA1 promoter: GUS construct was introduced into $P D A 1^{+}$strain 77-2-3. A single-spored isolate of the transformant was grown in Barz medium for $24 \mathrm{~h}$ and transferred to phosphate buffer with $100 \mu \mathrm{M}$ pisatin (filled symbols) or without pisatin (open symbols). Mycelial samples were taken at the specified times after pisatin addition and used to determine pisatin demethylase enzyme activity (normalized to $\mathrm{mg}$ [wet weight] of mycelia) in the living mycelia or GUS activity (normalized to $\mathrm{mg}$ of protein) in mycelial extracts taken from the same sample. 
GUS activity in the absence of pisatin but with nutrients at 8 $\mathrm{h}$ was 0.21 and $0.056 \mathrm{pmol} / \mathrm{min} / \mathrm{mg}$ for $278 \mathrm{mM}$ glucose and $166 \mathrm{mM}$ casamino acid treatments, respectively. GUS activity in transformants of the $\mathrm{Pda}^{-}$strain (94-6-1) carrying the $P D A 1:: G U S$ fusion was also found to peak at approximately the same time in the pisatin-treated culture and showed similar pisatin induction, glucose repression, and amino acid repression (Fig. 3). This strain proved to be easier to transform than 77-2-3. The results indicate that this promoter fusion construct is regulated in a manner similar to the native $P D A 1$ gene and so the $1.1-\mathrm{kb}$ promoter fragment appears to contain the necessary regulatory elements for pisatin, glucose, and amino acid regulation. Since regulation was similar in the $\mathrm{Pda}^{+}$and $\mathrm{Pda}^{-}$strains, the $\mathrm{Pda}^{-}$strain was used for further studies to avoid artifacts from competition for transcription factors or gene silencing between the native PDA1 gene and the introduced PDA1::GUS.

\section{Nutritional regulation of $P D A 1$.}

In order to determine the interdependence of pisatin and nutritional regulation, and to quantify the levels of glucose or amino acids required for repression, expression from the full- length PDA1 promoter::GUS construct was measured over varying concentrations of each nutrient (Fig. 3). Increasing nutrient concentrations suppressed GUS activity in the presence of pisatin, as expected from earlier studies of Pda enzyme activity. However, increasing nutrients also suppressed promoter activity in the absence of pisatin. Thus, nutritional repression was evident in the absence of pisatin induction. Pisatin inducibility was also observed in both the absence and presence of nutritional repression (Fig. 3). Thus, the two types of regulatory signals appear to act independently in their regulation of the $P D A 1$ promoter rather than through mutual interference.

The range of nutrient concentrations that reduced expression of the full-length PDA1 promoter was 1 to $55 \mathrm{mM}$ glucose and 1 to $4 \mathrm{mM}$ casamino acids. The highest concentrations in this range produced approximately 10-fold repression, compared with the control in phosphate buffer alone. The addition of non-metabolizable glucose analogs glucosamine and 2-deoxy-glucose (at $270 \mathrm{mM}$ ) repressed PDA1::GUS expression similarly to glucose (results not shown). Substitution of sucrose for glucose demonstrated an approximately twofold decrease in the threshold for repres-
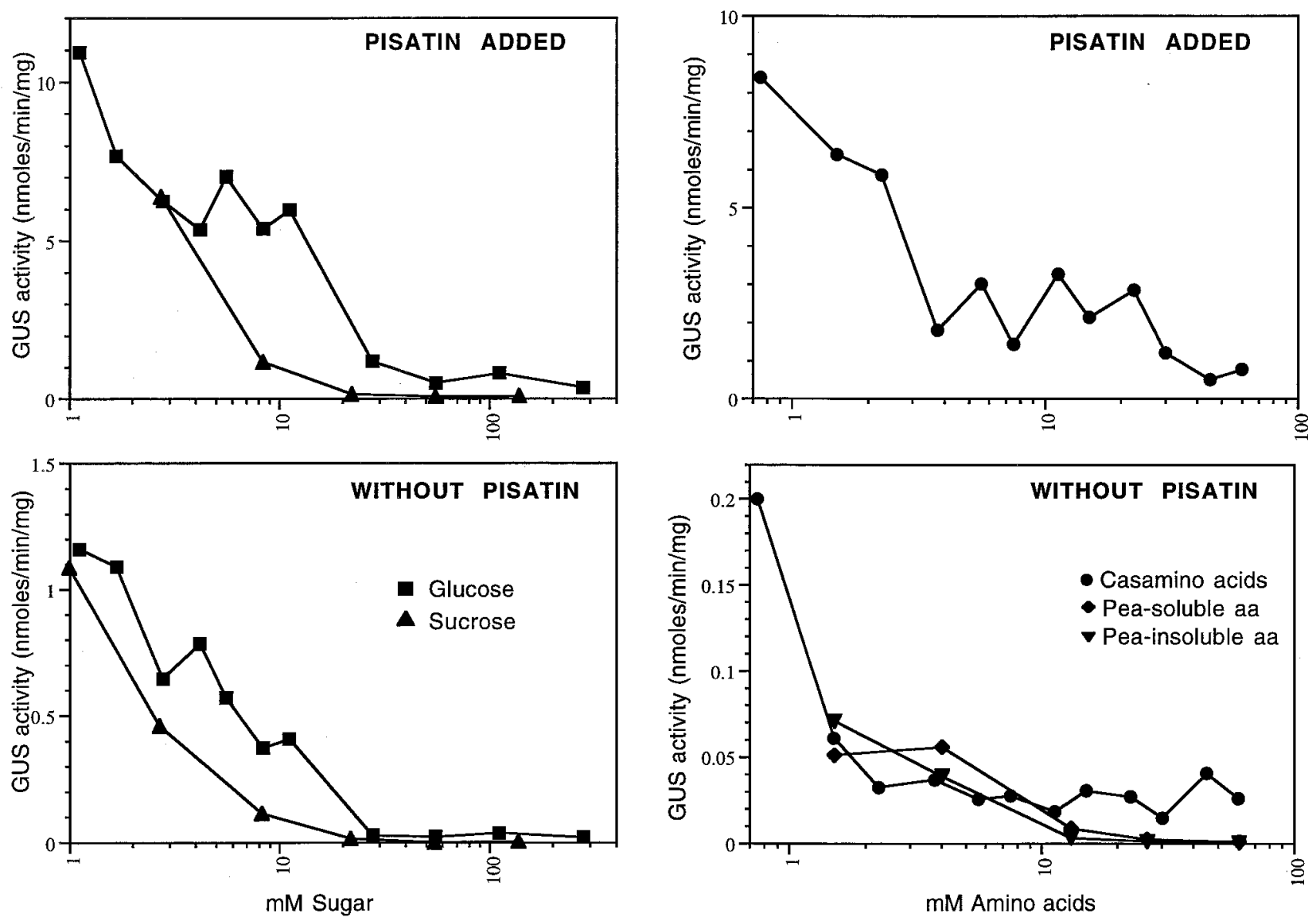

Fig. 3. Glucose and amino acid repression of $\beta$-glucuronidase (GUS) activity from the full-length PDA1 promoter. Transformant (14B1) of strain 94-6-1 carrying the full-length PDA1 promoter::GUS was grown for $24 \mathrm{~h}$ in Barz medium and the mycelia transferred to phosphate buffer. Differing amounts of nutrients (sugars left, amino acids right) were added to phosphate buffer upon transfer, with (top) or without (bottom) $100 \mu \mathrm{M}$ pisatin. After a further $8 \mathrm{~h}$ of incubation, required for pisatin induction, GUS activity in each treatment was measured. Points are the average of two experiments. Amino acid mixtures used: Bacto casamino acids, acid-soluble amino acids of pea stem, and acid-insoluble amino acids (proteins) from pea cotyledon. The latter two were mixed from separate amino acids in the molar ratios reported by Bar-Nun and Poljakoff-Mayyber (1979). GUS activity background level in untransformed $94-6-1$ is $3.2 \mathrm{pmol} / \mathrm{min} / \mathrm{mg}$. 
sion (Fig. 3). Mixtures of amino acids different from that present in the casamino acids but representing the amino acid composition of acid-soluble amino acids in pea cotyledon, or acid-insoluble (protein) amino acids in pea cotyledon (Bar-Nun and Poljakoff-Mayyber 1979) all gave threshold concentrations of repression similar to those of casamino acids (Fig. 3).

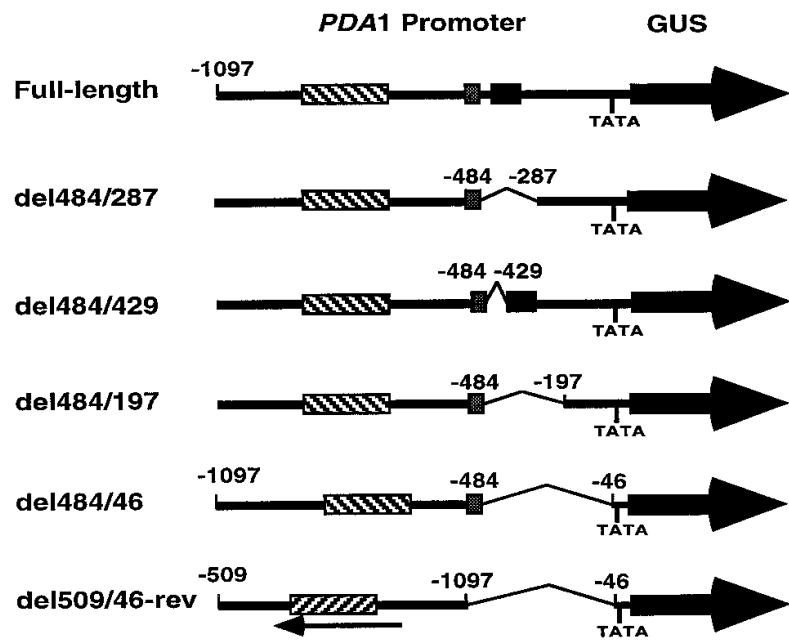

Fig. 4. $P D A 1$ promoter deletion constructs. Deletions in the $P D A 1$ promoter were constructed in PDA1::GUS through long-range polymerase chain reaction amplification. Endpoints of the internal deletions are shown. Numbers are relative to the site of transcription. Boxes are included for reference and correspond, left to right, as a general activation region (hatched), a pisatin-responsive element defined previously in vitro, and a nutritional response region defined in this study.
Identification of functional regions in the $P D A 1$ promoter that respond to pisatin, glucose, and amino acids in culture.

A series of deletions was made in the PDA1 promoter with long-range PCR originating from divergent primers complementary to the PDA1 promoter (Materials and Methods; Fig. 4). These deletion constructs were introduced into strain 94-61 through transformation. Single-spored transformants showing both stable hygromycin resistance and intact $P D A 1:$ GUS regions (confirmed by PCR amplification in chromosomal DNA isolated from the transformants) were further characterized. GUS activity was measured in transformants to quantify pisatin induction, glucose repression, and amino acid repression of the constructs. To account for the positional effect of the ectopic insertions, multiple independent transformants were assayed (Fig. 5). Variability in the basal level of GUS activity was observed between different transformants and within replicates of one transformant in separate experiments. Generally, pisatin inducibility was lower in transformants displaying high basal expression levels, whereas the extent of glucose or amino acid repression was decreased in transformants displaying low levels of basal expression. These effects are presumably due to either the limits of detection of a wide range in GUS activity, or intrinsic limitations in the cell's ability to modulate promoter activity to extremes above or below basal rates.

Initial deletions paralleled ones previously tested in an in vitro transcription assay (Ruan and Straney 1996). These constructs deleted the upstream terminal $300 \mathrm{bp}$ of the $P D A 1$ promoter fragment, a region that in vitro transcription studies indicated to be dispensable for full expression. Surprisingly, all deletions removing the promoter region between -1097 and -509 displayed no measurable GUS activ-

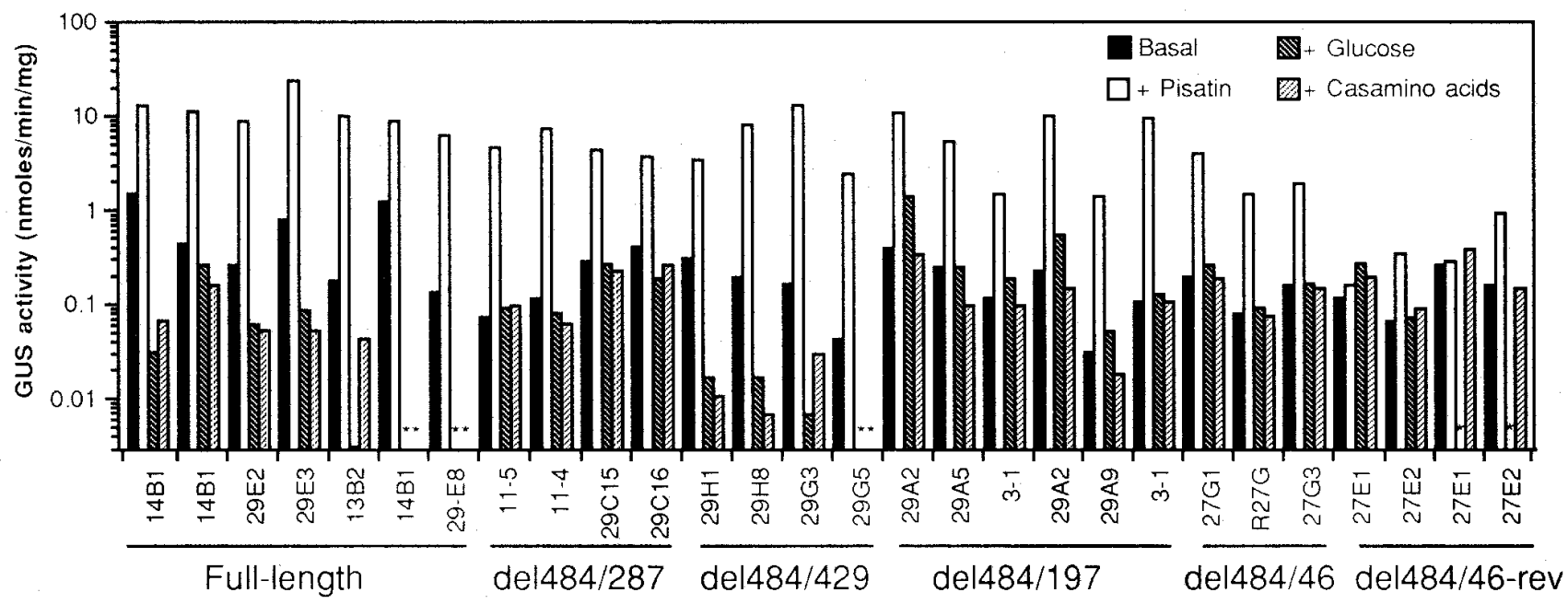

Fig. 5. Effect of promoter deletions upon response to regulatory signals in culture. $\beta$-Glucuronidase (GUS)-specific activity is shown for transformants of strain 94-6-1 carrying different PDA1::GUS constructs. Independent transformed strains and independent replicates of certain strains are grouped above the deletion construct used for transformation. Mycelial extracts used for the GUS assay were collected after incubation of mycelia for $8 \mathrm{~h}$ in phosphate buffer alone (Basal) or with the following treatments in phosphate buffer: $100 \mu \mathrm{M}$ pisatin (+ Pisatin), $278 \mathrm{mM}$ glucose (+ Glucose), or 142 mM casamino acids (+ Casamino acids). Treatments not tested are shown with an asterisk (*). Background GUS activity (untransformed 94-6-1) was measured in two samples with three replicates for each treatment and control; no significant difference was observed between conditions. Mean background GUS activity of 3.2 (SE 0.3 ) $\mathrm{pmol} / \mathrm{min} / \mathrm{mg}$ was subtracted from all measurements shown. A one-tailed paired $t$ test was performed for all measurements with each group bearing the same construct to compare treatment and basal activities. Significant $(P<0.05)$ differences between the means were found in the following comparisons: pisatin treatment vs basal activity-full-length, del484/287, del484/429, del484/197, del484/46; glucose treatment vs basal activity—full-length, del484/429; casamino acid treatment vs basal activity—full-length, del484/429. 
ity in the presence or absence of pisatin. Since this upstream region was essential for obtaining any measurable activity in vivo, subsequent deletions removed sequences further downstream (Figs. 4 and 5). The del484/46 construct lacked glucose and amino acid repression but retained pisatin induction. Since this construct is missing the region located between positions -46 and -484 there was a strong indication that the nutritional response element(s) is present within this region and was separate from pisatin regulatory element(s). Regions were systematically deleted between -484 and -46 , as shown in Figure 4. Deletion of the promoter regions between positions -197 and -484 (de1484/197) or 287 and -484 (del484/287) removed both glucose and amino acid repression. These deletion constructs displayed basal levels (expression in phosphate buffer with no pisatin or nutrients) comparable to that of the full-length promoter. Deletion of an upstream portion of this region, between 429 and -484 (del484/429) did not affect repression by either glucose or amino acid. These results indicate that the nutritional response region resides between positions -287 and -429 upstream of the transcriptional initiation site. Although these constructs map fairly large regions of the promoter, they produced $P D A 1$ promoter variants that lack nutritional repression but retained near normal pisatin induction. The deletion of the above region and the previously defined pisatin-responsive region with an inversion of the upstream region (del509/46-rev) produced a promoter that was constitutively expressed in culture (Fig. 5). This construct displayed normal basal level GUS activity in phosphate buffer but lacked pisatin inducibility as well as nutrient repression. Constitutive expression was confirmed in hourly measurements over a 12 -h time course in culture with pisatin (results not shown).

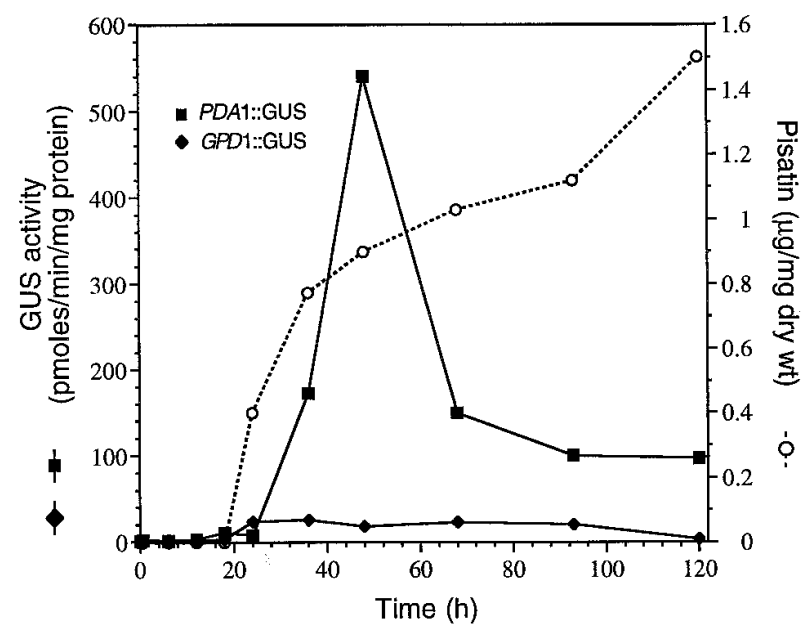

Fig. 6. $\beta$-Glucuronidase (GUS) expression from PDA1::GUS constructs during growth in pea epicotyl. Nectria haematococca strain 94-6-1 transformants carrying either a full-length PDA1::GUS fusion (14B1) or a constitutive GPD1::GUS fusion were inoculated separately onto wounded pea epicotyls as a spore suspension. Epicotyls were harvested at varying times after inoculation. Protein extracts from the infected epicotyls were assayed for GUS-specific activity, which was normalized to the protein contained in the epicotyl extract. Pisatin was assayed in samples of the same epicotyls by thin-layer chromatography and was normalized to the dry weight of the infected pea tissue extracted.

\section{Promoter regulation during plant pathogenesis.}

Infection of pea epicotyls with transformants of strain 94-61 carrying the full-length $P D A 1$ promoter::GUS fusion construct displayed GUS activity that peaked at $42 \mathrm{~h}$ after inoculation. This induction was coincident with the production of pisatin by the pea plant under these conditions (Fig. 6). A transformant of the highly virulent strain 77-2-3 carrying the same construct displayed approximately 10 -fold greater expression of GUS activity but with a similar pattern of GUS induction in planta (results not shown). For comparison, the constitutive GPD1 promoter from Cochliobolus heterostrophus (Van Wert and Yoder 1992) was used to construct a GPD1::GUS fusion. A transformant of 94-6-1 carrying this construct displayed neither pisatin induction nor amino acid repression in culture; however, it did display a fivefold induction by glucose in culture. When the transformant carrying the GPD1::GUS fusion was inoculated into pea epicotyls, GUS activity showed little modulation (Fig. 6). Thus, the modulation of GUS activity with the PDA1::GUS construct appears to be due to gene induction during pathogenesis, potentially in response to pisatin.

To directly evaluate the role of different response elements regulating PDA1 gene expression during pea pathogenesis, transformants bearing deletion constructs with different regulatory responses characterized in culture were used to infect pea. Pea epicotyls were inoculated with spores from the transformants carrying the full-length, del484/287, del484/46, and del509/46-rev PDA1::GUS constructs. The infected epicotyls were collected over a period of time. A cell-free extract from each sample was used for measuring the GUS activity. The amount of GUS activity was normalized to the amount of protein extracted. Differences in the amount of fungal biomass between samples, due to growth over time or variation in infection between pea seedlings, were accounted for by normalizing the GUS-specific activities to the relative amounts of fungal DNA and pea DNA extracted in the same cell-free extract. Thus, any modulation of expression levels observed in planta should represent changes in regulated expression rather than fungal growth. Fungal DNA in the extracts was measured by Southern analysis and hybridization with a fungal-specific rDNA probe. Pea DNA content in the extracts was measured by hybridization with a pea-specific rDNA probe. Neither probe displayed hybridization with control DNA from the other organism. GUS activity peaked at $48 \mathrm{~h}$ in pea tissues infected with a transformant bearing the full-length $P D A 1$ promoter in two independent experiments (Fig. 7). The del484/287 and del484/46 PDA1::GUS constructs, which lack nutritional repression but retain pisatin inducibility in culture, displayed modulation generally similar to that of the fulllength promoter; however, in all but one instance, the activities peaked earlier than with the full-length construct (Fig. 7). Peak GUS activity was reduced by approximately $10 \%$ in del484/287 and by approximately $40 \%$ with the larger deletion del484/46 in one experiment, but each produced greater expression (33 to 55\%) than the full-length promoter in an independent replicate of the experiment. Transformants with the construct del509/46-rev, which is largely pisatin insensitive in culture, displayed little modulation over the entire time course in both experiments. Thus, the induction of the PDA1 promoter during growth in pea was correlated strongly to its pisatin inducibility in culture, and not to its nutritional regulation. 


\section{DISCUSSION}

The PDA genes of $N$. haematococca provide a system to study the potential complexity of virulence gene regulation during pathogenesis, since both host-specific (pisatin) and non-specific (nutritional) signals have been identified that regulate the genes in culture. The presence of the highly pisatin-inducible $P D A 1$ gene in all pea-pathogenic field strains (Hirschi 1994; Miao et al. 1991) would suggest that pisatin inducibility may be a key trait during pathogenesis. We have used modifications of the PDA1 promoter in GUS fusion constructs to correlate regulation seen in culture, where con-
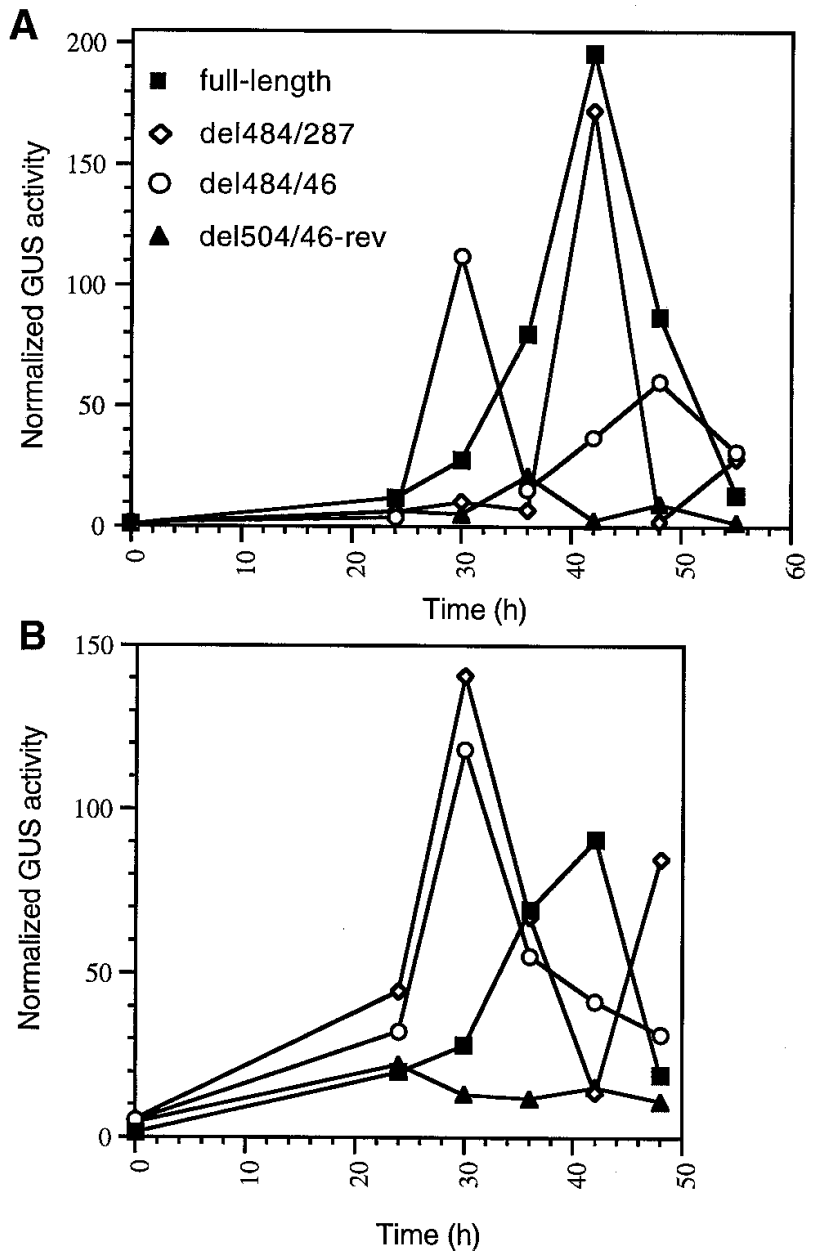

Fig 7. $\beta$-Glucuronidase (GUS) expression from altered $P D A 1$ promoters during growth in pea epicotyl. GUS activity was measured in pea epicotyls inoculated with transformants of strain 94-6-1 bearing the indicated $P D A 1$ promoter construct with altered nutritional and/or pisatin responses summarized in Figure 5. A, Results of one experiment. B, A second independent experiment with the same strains. Epicotyls were inoculated with spores, and at the times indicated three epicotyls undergoing each treatment were collected together to make a cell-free mycelial extract. These mycelial extracts were used for both GUS activity measurements and Southern blotting with fungal- and pea-specific rDNA probes. The normalized GUS activity is the GUS-specific activity of each sample ( $\mathrm{pmol} / \mathrm{min} / \mathrm{mg}$ of protein) multiplied by the ratio of fungal DNA (ng) to pea DNA $(\mu \mathrm{g})$ measured in that sample, to account for differences in fungal biomass during growth. Transformants used for inoculation: 14B1 (full-length), 11-4 (del484/287), 27G1 (del484/46), and 27E2 (del509/46-rev). ditions are easily controlled and defined, to regulation seen during pea pathogenesis. This allowed comparison of the contributions of each regulatory response toward the overall expression during pathogenesis. The demonstration that pisatin may be the dominant stimulus of $P D A 1$ during growth of $N$. haematococca on pea is significant in demonstrating the biological importance of regulation by host-specific cues.

The $P D A 1$ promoter displays nutritional regulation similar to that of a number of other genes characterized in plantpathogenic fungi. Glucose repression of PDA1 is similar to that observed for pectate lyase, cutinase, and two ipi genes (Gonzalez-Candelas and Kolattukudy 1992; Lin and Kolattukudy 1978; Pieterse et al. 1994). Nitrogen repression of PDA1 is limited to repression by amino acids (Straney and VanEtten 1994), similar to that of the laccase gene of $C$. parasitica (Larson and Nuss 1994), but it is not sensitive to inorganic nitrogen sources reported to inhibit $A V R 9$ of C. fulvum (Van den Ackerveken et al. 1994), MPG1 (Lau and Hamer 1996; Talbot et al. 1993) and host necrosis-associated proteins in $M$. grisea (Talbot et al. 1997). Nitrogen regulation is particularly interesting since nitrogen de-repression of $M P G 1$ is thought to directly stimulate $M P G 1$ expression in planta before the onset of full symptoms (Lau and Hamer 1996; Talbot et al. 1993). This is supported by the lack of both MPG1 nitrogen derepression in culture and expression in planta in two regulatory mutants (Lau and Hamer 1996). These observations have led to the proposal that nutritional starvation is a general cue for induction of pathogenicity genes (Talbot et al. 1997). By analogy to these examples, the nutrient regulation of PDA1 suggests that it too could be modulated in planta solely through encountering decreasing levels of the nutrient (derepression) during pathogenesis. However, it is also possible that nutrient repression represents a laboratory artifact if the levels of nutrients required for repression are much higher than that encountered in the plant. The lack of previous studies on the nutrient levels required for repression of $P D A 1$ or most other fungal virulence genes has made it difficult to predict the role of nutritional regulation during pathogenesis.

Using the $P D A 1::$ GUS reporter, we were able to determine the levels of these nutrients required to produce $P D A 1$ repression in culture. PDA1::GUS expression was partially suppressed by glucose or sucrose concentrations between 1 and $55 \mathrm{mM}$. These levels are within the range of that reported for sugars in pea epicotyl tissue (1.5 to $3 \%$ [wt/fresh wt] glucose, approximately $80 \mathrm{mM}$; Singh and MacLachlan 1983) or in the apoplast ( $9.8 \mathrm{mM}$ sucrose; Estruch et al. 1989). These sugar concentrations would be expected to produce full and partial repression of $P D A 1$ expression in culture, respectively. Amino acid repression acted over a range of 1 to $4 \mathrm{mM}$ in culture. The response was similar with different mixtures of amino acids, representing that in commercial casamino acids, or those reported for free amino acid pools or proteins in pea cotyledons. This uniformity supports earlier experiments (Straney and VanEtten 1994) that suggest that amino acid regulation of $P D A 1$ is similar to general amino acid control in yeast rather than responding to a specific amino acid. Xylem fluids from pea contain 16 to $40 \mathrm{mM}$ amino acids (Rochat and Boutin 1991) and free amino acids pools in pea roots are reported to be $397 \mu \mathrm{mol} / \mathrm{g}$ (fresh wt), or approximately 397 mM (Bar-Nun and Poljakoff-Mayyber 1979). Thus, free amino acid concentrations in certain compart- 
ments of pea would be expected to be sufficiently high to repress $P D A 1$ expression.

The above comparisons indicate that nutritional control of $P D A 1$ at physiologically relevant concentrations of sugars or amino acids can potentially reduce expression of $P D A 1$. However, the lack of detailed information about how these nutrients are partitioned within the tissues and how they may change during pathogenesis leaves one unable to predict a priori how the PDA1 gene would be regulated by these levels. Our GUS expression results demonstrate continued modulation of $P D A 1$ promoter activity during pathogenesis in the absence of nutritional regulation. This indicates that nutritional regulation alone is not inducing $P D A 1$ expression during pathogenesis, as would be expected if the pathogen experiences decreasing nutrient levels over this period. Variation in replicated experiments prevents us from quantifying a partial contribution of nutritional de-repression toward modulation; however, it appears to be relatively minor. Although the induction is apparently not caused by changes in nutrient levels, the earlier induction of the nutrient-insensitive promoter constructs would suggest that nutritional repression may delay PDA1 promoter expression.

Pisatin is the third potential regulator of PDA1 expression during pathogenesis, and is perhaps the most interesting because it represents a host-specific signal. Like other phytoalexins, pisatin is not present constitutively but is instead produced in response to infection. Thus, it is expected that this signal would rise during pathogenesis and stimulate $P D A 1$ expression. A previous PCR-based measurement of PDA1 expression during pathogenesis found $P D A 1 \mathrm{mRNA}$ to appear coincident with the induction of a plant gene encoding a pisatin biosynthetic enzyme (Hirschi and VanEtten 1996). Our measurement of GUS expression from the full-length PDA1 promoter::GUS fusion confirms this result and provides improved quantitation over the PCR-based detection. PDA1 expression increased with a 6-h lag after the appearance of pisatin in the infected pea, consistent with a 6- to 8-h lag observed in culture. The comparison of GUS expression in planta with the various promoter constructs displays strong correlation between pisatin inducibility of the promoter and induced $P D A 1$ expression in planta. All stable transformants carrying internal promoter deletions that retained high pisatin inducibility in culture displayed induced GUS activity in planta whether or not nutritional regulation was present. The loss of modulation in planta was only seen in the PDA1 construct that lacked pisatin responsiveness in culture. The lack of modulation in this construct appears to be specific to the loss of pisatin induction since the construct retained a normal basal level of activity in culture and during pathogenesis. These results indicate that pisatin produced by the host acts as the major stimulatory signal encountered by $N$. haematococca during pathogenesis of pea.

One concern in our analysis is that the strain used for deletion analysis (94-6-1) displays low virulence on pea, due to its lack of the $1.6 \mathrm{Mb}$ chromosome containing the PDA1 gene, and so may not represent events occurring during infection by highly virulent strains. This strain was selected for this study to avoid interference between the native $P D A 1$ gene and the introduced constructs, either through competition for transcription factors or sense suppression. The use of the low virulence strain also delays the necrosis of pea tissue, which may lead to difficulty in measuring GUS activity. At this point, the only difference between high- and low-virulence strains was a higher level of PDA1::GUS induction in planta and in culture in the former. It does not appear that other genes providing major regulation of PDA1 are on the same 1.6 Mb chromosome with PDA1 and so the 94-6-1 seems to be an adequate model. However, we cannot rule out that nutritional stimulation could occur if glucose and amino acid pools are depleted late in pathogenesis at stages that the low virulence isolate could not achieve.

Although the deletions presented in this work are not designed to define minimal regions of action, the results indicate their location and how they interact. One conclusion from these studies is that pisatin and nutritional regulatory pathways are independent. The increased sensitivity of the GUS reporter assays allowed detection of nutritional repression in the absence of pisatin; thus, nutrients do not simply interfere with pisatin inducibility. Further, each response is determined by separate regions of the $P D A 1$ promoter. Repression by both glucose and amino acids is restricted to a 142-bp region ( -287 to -429 ) that lacks any role in pisatin inducibility. This region corresponds to a negative-acting site characterized by in vitro transcription assays (Ruan and Straney 1996). Its role in glucose repression may be explained by the presence of two adjacent, inverted, consensus binding sites (SYGGRG) for CreA (Cubero and Scazzocchio 1994), a glucose repressor identified in Aspergillus nidulans. The independent action of glucose repression and pisatin induction of $P D A 1$ would be similar to that seen in the regulation proline-utilization genes by CreA and gene-specific regulators (Cubero and Scazzocchio 1994). It is in contrast to CREA action at the A. nidulans alcA promoter where CREA suppresses ethanol signaling by alcR through repression of alcR transcription and competition for alcR binding on the alcA promoter. (Kulmberg et al. 1993). The amino acid regulation of this region is more difficult to explain from its sequence. It contains no predicted binding sites for cross-pathway, amino acid-responsive activators such as GCN4 of Saccharomyces cerevisiae and CPC-1 of Neurospora crassa and C. parasitica (Arndt and Fink 1986; Ebbole et al. 1991; Wang et al. 1998). Regulation of PDA1 may instead be similar to that of genes in $N$. crassa that are induced by amino acid starvation but are independent of CPC-1 (Flint 1985).

The pisatin-responsive regulatory elements of $P D A 1$ appear to be more complex than the nutritional regulatory site. Previous in vitro binding and transcription assays identified a pisatin-responsive element that lies in a 13-bp sequence at positions -494 to -507 (He et al. 1996; Ruan and Straney 1996). In contrast, the in vivo studies presented here indicate that pisatin inducibility is associated with a region further upstream $(-509$ to -1097$)$ that is required for obtaining any promoter activity in vivo. The findings from this study that point to pisatin as a primary regulatory signal support the need for further definition of the pisatin-responsive region that acts in vivo.

\section{MATERIALS AND METHODS}

\section{Fungal strains.}

N. haematococca MPVI strains 94-6-1 and 77-2-3 was used for transformation. 94-6-1 is a $\mathrm{Pda}^{-}$progeny of a backcross 
between the PDA1 containing strain 77-2-3 and the $\mathrm{Pda}^{-}$strain 44-100 (Kistler and VanEtten 1984). Strains were maintained on V8 media slants. Media, growth conditions, and PDA1 induction were similar to those described earlier (Kistler and VanEtten 1984).

\section{Generation of fusion construct and promoter deletions.}

The ATG start codon of PDA1 $(+110$ relative to the start site of transcription) is in an $N s p C I$ site. An $N s p C 1$ restriction fragment containing the $1.1-\mathrm{kb}$ promoter fragment from $\mathrm{pDA} 1$ (Straney and VanEtten 1994) was ligated into the NspCIcompatible SphI site in the pBS+ (Stratagene, La Jolla, CA) cloning vector. The HindIII site 8 bp downstream of the SphI site in the pBS+ polylinker was converted into an NheI site through filling of the HindIII site and religating. The NheI/KpnI fragment containing the PDA1 promoter was then inserted into an $X b a \mathrm{I} / K p n \mathrm{I}$-digested pBluescript $\mathrm{KS}+$ (Stratagene, La Jolla, CA) derivative of pBI101 (Clonetech, Palo Alto, CA). This creates a translational fusion of the $P D A 1$ promoter with the GUS gene::Agrobacterium tumifaciens NOS terminator from $\mathrm{pBI} 101$ and adds 12 amino acids to the GUS translated product encoded by polylinker sequences (Fig. 1). For production of a selectable marker, we made a pBluescript $\mathrm{KS}+$ derivative of $\mathrm{pDH} 33$, containing the hygromycin phosphotransferase gene $(\mathrm{Hyg} \mathrm{B})$ fused to a 300 bp MluI/ClaI fragment of the Aspergillus niger glaA promoter (Fowler et al. 1990) and the Aspergillus nidulans trpC terminator. An EcoRI/BstXI fragment of this selection construct was ligated into downstream EcoRI/BstXI sites present in the $P D A 1::$ GUS. The resulting construct, $P D A 1::$ GUS, contains two sets of fusion: a PDA1::GUS::NOS terminator and a gla $\mathrm{A}:$ :hygB::trpC terminator.

The GPD1::GUS fusion was constructed by ligation of the $K p n I / S a l$ I fragment containing the GPD1 promoter from p41:GPDa (Van Wert and Yoder 1994) into a SalI/KpnIdigested pBluescript KS+ derivative of pBI101 described above. This creates a translational fusion of the GPD1 promoter to the GUS::NOS terminator. The EcoRI/BstXI cassette containing the glaA promoter::HygB::trpC terminator fusion was ligated to a EcoRI/ BstXI-digested GPD1::GUS construct to create the final construct used for transformation.

Deletions in the PDA1 promoter were generated by designing divergent PCR primers flanking the region to be deleted. These primers were oriented such as to amplify the entire plasmid except the sequence to be deleted and included a KpnI site added to the $5^{\prime}$ end. A derivative of the PDA1::GUS plasmid in Figure 1, in which the KpnI site was destroyed by $3^{\prime}$ end digestion and religation, was used for amplification by long-range PCR (GeneAmp XL-PCR; Perkin Elmer, Branchburg, NJ) according to the manufacturer's instructions. After purification by electrophoresis on an agarose gel, the PCR product was digested with KpnI, self ligated, and transformed into the Escherichia coli strain DH5 $\alpha$. The transformed colonies were selected by their resistance to ampicillin. The deletion of the appropriate sequences and the integrity of remaining sequences were confirmed by sequencing.

\section{N. haematococca transformation.}

$N$. haematococca was transformed by the method described by Wasmann and VanEtten (1996) with the exception that each transformation used $10^{7}$ protoplasts mixed with $10 \mu \mathrm{g}$ of salmon sperm DNA, $1 \mathrm{mM}$ spermidine, $10 \mu \mathrm{g}$ of plasmid, but no aurintricarboxylic acid. Transformants usually appeared in 4 to 7 days. Each transformant was single spored and then maintained on regeneration media slants supplemented with $35 \mu \mathrm{g}$ of hygromycin per ml. DNA was isolated from each transformant and used in a PCR screen with the GUS sequencing primer (5'-TCACGGGTTGGGGTTTTC) and the M13 Rev primer to confirm that the promoter-GUS fusion was not interrupted. Southern hybridization was performed on $K p n I / E c o$ RI digests of genomic DNA from transformants separated on a $1 \%$ agarose gel and transferred to a Nytran membrane. These filters were probed with a ${ }^{32} \mathrm{P}$-labeled GUS gene fragment.

\section{Mycelial extracts and GUS assay.}

The transformants were grown in Barz media for $24 \mathrm{~h}$ at $28^{\circ} \mathrm{C}$ with shaking at $250 \mathrm{rpm}$ (series 25; New Brunswick, Edison, NJ). The mycelia were harvested, suspended in phosphate buffer at $30 \mathrm{mg}$ (wet weight)/ml and treated with $0.1 \mathrm{mM}$ pisatin, $5 \%$ glucose or $0.8 \%$ Bacto casamino acids (Difco, Detroit, MI), unless other concentrations are given. Treatment with other mixtures of amino acids used the mole percents reported in Bar-Nun and Poljakoff-Mayyber (1979). After $8 \mathrm{~h}$ of treatment, the mycelia were harvested by filtration, lyophilized, and ground in liquid nitrogen with a mortar and pestle. The powdered mycelia were suspended in GUS extraction buffer $\left(50 \mathrm{mM} \mathrm{NaPO}_{4} \mathrm{pH} 7.0,10 \mathrm{mM}\right.$ $\mathrm{Na}_{2}$ EDTA, $0.1 \%$ each of Sarkosyl and Triton X 100, $1 \mathrm{mM}$ phenylmethyl-sulfonyl fluoride and $10 \mathrm{mM} \beta$-mercaptoethanol). After centrifugation in a microcentrifuge for $5 \mathrm{~min}$, the supernatant was collected and total protein was measured with a Bradford protein assay (BioRad, Hercules, CA). All GUS assays used the MUG (4-methylumbelleriferyl- $\beta$ D-glucuronide) fluorescence method of Jefferson (1987) with the addition of $20 \%$ methanol in the assay buffer to reduce background GUS activity in pea samples. The relative fluorescence of the product was measured on a Farrand fluorometer and was used to calculate the GUS activity from the slope of four time points and 4-methylumbelliferone standards. Where appropriate, Pda enzyme activity was measured in living mycelia of transformants by the method of Kistler and VanEtten (1984).

\section{Pea infection.}

Macroconidia from $N$. haematococca transformants grown on regeneration medium (Wasmann and VanEtten 1996) with $35 \mu \mathrm{g}$ of hygromycin per $\mathrm{ml}$ were washed twice and suspended in distilled water at $5 \times 10^{7}$ spores per ml. Four-dayold Alaska pea (Vermont Bean Seed, Fairhaven, VT) seedlings, germinated from surface-disinfected seeds and grown in the dark on cheesecloth soaked with Hoagland's solution, were wounded by aseptically slicing the length of the epicotyl with a scalpel. After wounding, the seedlings were dipped in the above spore suspension and the seedlings were incubated on top of five layers of cheesecloth wetted with $1 / 20 \times$ Hoagland's solution and contained within closed boxes in the dark at $18^{\circ} \mathrm{C}$. At the times given after inoculation, the epicotyl was harvested, lyophilized, and ground in liquid nitrogen. The powdered epicotyl was suspended in approximately 3 volumes of GUS extraction buffer. GUS activity in the extract was assayed the same as described above for mycelial samples. 


\section{Southern hybridization to estimate fungal biomass in pea tissue.}

The same infected seedling extracts used for GUS activity measurements were used for fungal and plant DNA quantitation. An aliquot of the sample in GUS extraction buffer was extracted once each with phenol, phenol-chloroform, and chloroform, and then was ethanol precipitated. For analysis of fungal DNA, the resuspended sample DNA was restricted with AvaI, electrophoresed on a $0.8 \%$ agarose gel, and transferred to a Nytran filter (Schleicher \& Schuell, Keene, NH). The filters were probed with a 535-bp PCR product amplified from $N$. haematococca DNA representing the internal transcribed spacer sequence (ITS) of the rRNA repeat (O'Donnell and Gray 1995) (primers: ITS1, TCCGTAGGTGAACCTGC GG; NITS1a, 5'-GAGTTGGGTGTTTTACG) and were washed under stringent conditions, as specified by manufacturer directions. The expected 750-bp AvaI band was quantitated on a phosphoimager (Molecular Dynamics, Sunnyvale, CA). Intensities of bands were used to estimate DNA content by comparison with fungal DNA standards of known quantities on each blot. One-third the volume of the same DNA sample was assessed for pea DNA content by attachment to Nytran filters through a slot-blot apparatus. These filters were probed with a 170-bp rDNA probe derived from the sequence of pea ITS1 (O'Donnell and Gray 1995) (PCR amplified from primers: 5'-CATCGGAGGGGAACGACAA; 5'-TCCGTTG CCGAGAGTCATTTTAT) and were washed under stringent conditions, as above. Intensities were converted to quantities of pea DNA by comparison with uninfected pea DNA standards of known concentration.

\section{ACKNOWLEDGMENTS}

We are grateful to S. Van Wert and O. C. Yoder for plasmid p41::GPDa and D. Cullen for pDH33. This research was supported by a grant from the National Science Foundation (MCB-9419266).

\section{LITERATURE CITED}

Arndt, K., and Fink, G. R. 1986. GCN4 protein, a positive transcription factor in yeast, binds general control promoters at all 5' TGACTC3' sequences. Proc. Natl. Acad. Sci. USA 83:8516-8520.

Bar-Nun, N., and Poljakoff-Mayyber, A. 1979. Intervarietal differences in the amino acid composition of pea roots as related to their response to salinity. Ann. Bot. 44:309-314.

Benito, E., Prins, T., and van Kan, J. 1996. Application of differential display RT-PCR to the analysis of gene expression in a plant-fungus interaction. Plant Mol. Biol. 32:947-957.

Ciuffetti, L. M., and VanEtten, H. D. 1996. Virulence of a pisatin demethylase-deficient Nectria haematococca MVI isolate is increased by transformation with a pisatin demethylase gene. Mol. PlantMicrobe Interact. 9:787-792.

Cubero, B., and Scazzocchio, C. 1994. Two different, adjacent and divergent zinc finger binding sites are necessary for CREA-mediated carbon catabolite repression in the proline gene cluster of Aspergillus nidulans. EMBO J. 13:407-414.

Ebbole, D. J., Paluh, J. L., Plamann, M., Sachs, M. S., and Yanofsky, C. 1991. cpc-1, the general regulatory gene for genes of amino acid biosynthesis in Neurospora crassa, is differentially expressed during the asexual life cycle. Mol. Cell Biol. 11:928-934.

Ehrenshaft, M., and Upchurch, R. G. 1993. Host protein(s) induces accumulation of the toxin cercosporin and mRNA in a phytopathogenic strain of Cercospora kikuchii. Physiol. Mol. Plant Pathol. 43:95-107.

Estruch, J. J., Pereto, J. G., Vercher, Y., and Beltran, J. P. 1989. Sucrose loading in isolated veins of Pisum sativum: Regulation by abscisic acid, gibberellic acid and cell turgor. Plant Physiol. 91:259-265.
Flint, H. J. 1985. Changes in gene expression elicited by amino acid limitation in Neurospora crassa strains having normal or mutant cross-pathway amino acid control. Mol. Gen. Genet. 200:243-290.

Fowler, T., Berka, R. M., and Ward, M. 1990. Regulation of the glaA gene of Aspergillus niger. Curr. Genet. 18:537-545.

Gonzalez-Candelas, L., and Kolattukudy, P. E. 1992. Isolation and analysis of a novel inducible pectate lyase gene from the phytopathogenic fungus Fusarium solani f. sp. pisi (Nectria haematococca, mating population VI). J. Bacteriol. 174:6343-6349.

Guo, W., Gonzalez-Candelas, L., and Kolattukudy, P. E. 1996. Identification of a novel pelD gene expressed uniquely in planta by Fusarium solani $\mathrm{f}$. sp. pisi (Nectria haematococca, mating type VI) and characterization of its protein product as an endo-pectate lyase. Arch. Biochem. Biophys. 332:305-312.

He, J., Ruan, Y., and Straney, D. 1996. Analysis of determinants of binding and transcriptional activation of the pisatin-responsive DNA binding factor of Nectria haematococca. Mol. Plant-Microbe Interact. 9:171-179.

Hirschi, K., and VanEtten, H. 1996. Expression of the pisatin detoxifying genes (PDA) of Nectria haematococca in vitro and in planta. Mol. Plant-Microbe Interact. 9:483-491.

Hirschi, K. D. 1994. Partial characterization of the PDA genes in Nectria haematococca MPVI. Ph.D. thesis. University of Arizona, Tucson.

Jefferson, R. A. 1987. Assaying chimeric genes in plants: The GUS gene fusion system. Plant Mol. Biol. Rep. 5:387-405.

Kasahara, S., and Nuss, D. L. 1997. Targeted disruption of a fungal Gprotein $\beta$ subunit gene results in increased vegetative growth but reduced virulence. Mol. Plant-Microbe Interact. 10:984-993.

Kistler, H. C., and VanEtten, H. D. 1984. Regulation of pisatin demethylation in Nectria haematococca and its influence on pisatin tolerance and virulence. J. Gen. Microbiol. 130:2605-2613.

Kulmberg, P., Mathieu, M., Dowzer, C., Kelly, J., and Felenbok, B. 1993. Specific binding sites in the alc $\mathrm{R}$ and alc $\mathrm{A}$ promoters of the ethanol regulon for the CREA repressor mediating carbon metabolite repression in Aspergillus nidulans. Mol. Microbiol. 7:847-857.

Larson, T. G., and Nuss, D. L. 1994. Altered transcriptional response to nutrient availability in hypovirus-infected chestnut blight fungus. EMBO J. 13:5616-5623.

Lau, G., and Hamer, J. 1996. Regulatory genes controlling MPG1 expression and pathogenicity in the rice blast fungus Magnaporthe grisea. Plant Cell 8:771-781.

Lin, T. S., and Kolattukudy, P. E. 1978. Induction of a biopolyester hydrolase (cutinase) by low levels of cutin monomers in Fusarium solani f. sp. pisi. J. Bacteriol. 133:942-951.

Miao, V. P. W., Matthews, D. E., and VanEtten, H. D. 1991. Identification and chromosomal locations of a family of cytochrome P-450 genes for pisatin detoxification in the fungus Nectria haematococca. Mol. Gen. Genet. 226:214-223.

Mitchell, T. K., and Dean, R. A. 1995. The cAMP-dependent protein kinase catalytic subunit is required for appressorium formation and pathogenesis by the rice blast pathogen Magnaporthe grisea. Plant Cell 7:1869-1878.

O’Donnell, K., and Gray, L. E. 1995. Phylogenetic relationships of the soybean sudden death syndrome pathogen Fusarium solani f. sp. phaseoli inferred from rDNA sequence data and PCR primers for its identification. Mol. Plant-Microbe Interact. 8:709-716.

Oeser, B., and Yoder, O. C. 1994. Pathogenesis by Cochliobolus heterostrophus transformants expressing a cutinase-encoding gene from Nectria haematococca. Mol. Plant-Microbe Interact. 7:282-289.

Pieterse, C. M. J., Derksen, A.-M. C. E., Folders, J., and Govers, F. 1994. Expression of the Phytophthora infestans ipiB and ipiO genes in planta and in vitro. Mol. Gen. Genet. 244:269-277.

Rochat, C., and Boutin, J.-P. 1991. Metabolism of phloem-borne amino acids in maternal tissues of fruit of nodulated or nitrate-fed pea plants (Pisum sativum L.). J. Exp. Bot. 42:207-214.

Ruan, Y., and Straney, D. C. 1996. Identification of elements in the PDA1 promoter of Nectria haematococca necessary for a high level of transcription in vitro. Mol. Gen. Genet. 250:29-38.

Schäfer, W., Straney, D., Ciuffetti, L., VanEtten, H. D., and Yoder, O. C. 1989. One enzyme makes a fungal pathogen, but not a saprophyte, virulent on a new host plant. Science 246:247-249.

Singh, R., and MacLachlan, G. 1983. Transport and metabolism of sucrose versus hexoses in relation to growth in etiolated pea stem. Plant Physiol. 71:531-535. 
Stahl, D. J., Theuerkauf, A., Heitefuss, R., and Schäfer, W. 1994. Cutinase of Nectria haematococca (Fusarium solani f. sp. pisi) is not required for fungal virulence or organ specificity on pea. Mol. PlantMicrobe Interact. 7:713-725.

Straney, D. C., and VanEtten, H. D. 1994. Characterization of the PDA1 promoter of Nectria haematococca and identification of a region that binds a pisatin-responsive DNA binding factor. Mol. Plant-Microbe Interact. 7:256-266.

Talbot, N. J., Ebbole, D. J., and Hamer, J. E. 1993. Identification and characterization of $M P G 1$, a gene involved in pathogenicity from the rice blast fungus Magnaporthe grisea. Plant Cell 5:1575-1590.

Talbot, N. J., McCafferty, H. R. K., Ma, M., Moore, K., and Hamer, J. E. 1997. Nitrogen starvation of the rice blast fungus Magnaporthe grisea may act as an environmental cue for disease expression development. Physiol. Mol. Plant Pathol. 50:179-195.

Van den Ackerveken, G. F. J. M., Van Kan, J. A. L., Joosten, M. H. A. J., Muisers, J. M., Verbakel, H. M., and De Wit, P. J. G. M. 1992. Characterization of two putative pathogenicity genes of the fungal tomato pathogen Cladosporium fulvum. Mol. Plant-Microbe Interact. 6:210215.

Van den Ackerveken, G. F. J. M., Dunn, R. M., Cozijnsen, T. J., Vossen,
P., Van den Broek, H. W. J., and De Wit, P. J. G. M. 1994. Nitrogen limitation induces expression of the avirulence gene $a v r 9$ in the tomato pathogen Cladosporium fulvum. Mol. Gen. Genet. 243:277-295.

Van Wert, S., and Yoder, O. 1994. Relative strengths of promoters from Cochliobolus heterostrophus. Curr. Genet. 25:217-222.

Van Wert, S. L., and Yoder, O. C. 1992. Structure of the Cochliobolus heterostrophus glyceraldehyde-3-phosphate dehydrogenase gene. Curr. Genet. 22:29-35.

VanEtten, H. D., and Barz, W. 1981. Expression of pisatin demethylase ability in Nectria haematococca. Arch. Microbiol. 129:56-60.

Wang, P., Larson, T. G., Chen, C.-H., Pawlyk, D. M., Clark, J. A., and Nuss, D. L. 1998. Cloning and characterization of a general amino acid control transcriptional activator from the chestnut blight fungus Cryphonectria parasitica. Fungal Genet. Biol. 23:81-94.

Wasmann, C. C., and VanEtten, H. D. 1996. Transformation-mediated chromosome loss and disruption of a gene for pisatin demethylase decrease the virulence of Nectria haematococca on pea. Mol. PlantMicrobe Interact. 9:793-803.

$\mathrm{Xu}$, J., and Hamer, J. 1996. MAP kinase and cAMP signaling regulate infection structure formation and pathogenic growth in the rice blast pathogen Magnaporthe grisea. Genes Dev. 10:2696-2706. 